of irrigation in the river valleys and dry farming in the zone where there is a minimum rainfall of 800 millimetres and a maximum of 1,200 millimetres. Extensive irrigation works have been put in hand, and research undertaken into the question of the varieties of cotton most suitable for cultivation by these two methods. At the same time factories have been constructed, the means of communication have been improved, and the education of the native producer has been taken in hand.

The new conditions will inevitably affect the basis of native society which in the last thirty years has already undergone such radical changes. Judging, however, from conditions in Senegal, where societies for encouraging the native producer, together with a system of agricultural credits, have been introduced, progress will continue to be made on lines which may be regarded as satisfactory from every point of view.

\title{
LA CONCEPTION DE L'ÂME CHEZ LES BANTOU
}

\section{PAR W. C. WILLOUGHBY}

$$
\text { (Voir p. 338) }
$$

Aucun Bantou n'a encore exposé ses propres conceptions religieuses et nous ne connaissons celles-ci que par des observations faites par des étrangers. Or il ne semble pas que ces conceptions puissent être indiquées sans tenir compte de la mentalité du peuple observé et nous ne sommes guère avertis de cette dernière. Les théories formulées et les explications données ne semblent pas caractériser très exactement les Bantou et leur état d'esprit. Ceux-ci vivent dans un milieu influencé par la magie, hanté par de formidables puissances occultes, ils sont embarrassés d'une science stérile manquant de règles pour prouver la légitimité de ses conclusions. Leur foi ne saurait donc être comparée à la nôtre, bien que leur intelligence ressemble à celle de l'Européen.

Les Bantou croient à l'existence de quelque chose de subtil dans l'homme, ils nomment ce quelque chose: souffle, vent, vapeur, ombre, c'est ce que nous appelons: âme, esprit, spectre; et cependant il est presque impossible de rendre exactement ces expressions dans une langue européenne. Il y a en Bantou un ajustement précis des termes pour l'âme incorporée et l'âme excorporée que nous soupçonnons rarement.

Le Bantou n'a pas abouti par une chaîne de déductions logiques à l'idée de l'âme intérieure. Cette conception est antérieure à la race bantoue, elle procède des impressions confuses du rêve, elle se fonde sur l'expérience, non sur la théorie. Le sommeil et la mort sont frère et sœur. L'âme, même éloignée du corps, reste en relation avec lui et après la mort l'esprit désincarné conserve aussi des relations avec ses restes terrestres.

L'observateur européen est enclin à se poser deux questions à propos de 
l'âme: naît-elle en même temps que le corps; est-elle impérissable? L'étude des rites magico-religieux révèle que dans la communauté bantoue les nouveau-nés sont solennellement présentés à celle-ci, et qu'on ne leur fait point de funérailles lorsqu'ils meurent avant cette cérémonie. On en a conclu que dans ce cas ils étaient considérés comme n'ayant pas encore d'âme. Par ailleurs on a noté que certaines âmes de personnes décédées sont susceptibles de renaître dans leurs descendants. Mais on peut être enclin à penser que l'âme est supposée de pénétrer dans le fœus lorsque celui-ci commence à s'agiter, bien qu'il y ait doute à ce sujet. En second lieu, et relativement à l'éternité de l'âme, on peut remarquer que l'on prophétise les défauts ordinaires seulement pendant trois générations, tandis qu'on vénère les chefs importants durant des siècles. Certaines coutumes suggèrent donc l'hypothèse que l'âme est censée de survivre longtemps après la mort, tandis que d'autres donnent à penser qu'elle est dépourvue de puissance après la disparition du corps.

La mort ne rompt pas tous les liens entre le corps et l'âme, celle-ci vit dans le tombeau, ou en habite les abords et visite ses parents chaque nuit. Ce n'est qu'après l'accomplissement de certains rites qu'elle gagne, après une période plus ou moins prolongée, les pays des esprits.

L'observation des rites funéraires, même quand le-corps est absent, suggère l'opinion que leur accomplissement est nécessaire pour que l'âme soit libérée.

Il ne semble pas douteux que le monde des esprits ne soit souterrain pour les Bantou, de nombreux exemples tirés du folk-lore le prouvent. L'association des morts avec les corps célestes ne démontre pas le contraire, comme on peut le croire, puisque le soleil et la lune passent la moitié du temps dans le monde inférieur. Celui qu'imaginent les Bantou est semblable à celui qu'ils habitent, et chacun y demeure avec son peuple pour cultiver la terre, faire paitre les troupeaux, chasser, s'adonner aux métiers et aux arts comme sur la terre. Et si le travail est si détesté des Bantou ainsi qu'on le prétend, comment n'imaginent-ils pas un lieu où l'humanité épuisée ne fasse rien, ne goûte rien, pendant l'éternité?

\section{LA POÉSIE DE L'AFRIQUE DE L'EST \\ PAR A. Werner$$
\text { (Voir p. 348) }
$$

ON peut distinguer dans l'Afrique orientale la poésie primitive des tribus intérieures et la poésie plus ou moins littéraire des Swahili. La première est en général étroitement liée à la danse et au son du tambour qui en scande la mesure. Elle a donc un certain rythme, mais aucune versification proprement dite. Quelques-uns de ces chants sont très anciens, ils ont une signification rituelle, fidèlement transmise de génération en génération et pourtant Dd 2 